doi: http://dx.doi.org/10.7124/bc.0009C9

\section{Selective inhibition of metastasis in vivo, partly through disruption of nucleoli}

K. J. Frankowski, C. Wang, S. Patnaik, F. J. Schoenen, N. Southall, D. Li, Y. Teper, W. Sun, I. Kandela, D. Hu, C. Dextras, Z. Knotts, Y. Bian, J. Norton, S. Titus, M. A. Lewandowska, Y. Wen, K. I. Farley, L. M. Griner, J. Sultan, Z. Meng, M. Zhou, T. Vilimas, A. S. Powers, S. Kozlov, K. Nagashima, H. S. Quadri, M. Fang, C. Long, O. Khanolkar, W. Chen, J. Kang, H. Huang, E. Chow, E. Goldberg, C. Feldman, R. Xi, H. R. Kim, G. Sahagian, S. J. Baserga, A. Mazar, M. Ferrer, W. Zheng, A. Shilatifard, J. Aubé, U. Rudloff, J. J. Marugan, $\underline{\text { S. Huang }}$

University of Kansas, KS. , Northwestern University, Chicago, IL, NIH, Bethesda, MD, Yale School of Medicine, New Haven, CT, , Leidos Biomedical Research, Inc., Frederick, MD, Tufts University, Boston, MA, UNC Eshelman School of Pharmacy, Chapel Hill, NC

s-huang2@northwestern.edu

Identification and development of effective anti-cancer drugs using PNC as a phenotypic marker for metastatic potential of cancer cells. Methods: To identify compounds selectively targeting the metastatic state, we used the perinuclear compartment (PNC), a complex nuclear structure associated with metastatic behaviors of cancer cells, as a phenotypic marker for a high-content screen of over 140,000 structurally diverse compounds. Extensive medicinal chemical optimization of a screening hit yielded metarrestin, which has been evaluated for in vitro and in vivo effi- cacy against xenograft tumor growth and metastasis from three type's human cancers in animal models. Biochemical and cellular characterizations have identified some of the modes of action for metarrestin. Results: Metarrestin disassembles PNCs in multiple cancer cell lines, inhibits invasion in vitro, blocks metastatic development in three mouse models of human cancer, and extends survival of mice in a metastatic pancreatic cancer xenograft model even when macrometastasis have developed. Metarrestin induces little toxicity or discernable adverse effects in animals when treated daily up to 4 months. Metarrestin selectively disrupts the nucleolar structure and inhibits RNA polymerase ( $\mathrm{Pol})$ I transcription in cancer cells, at least in part by interacting with the translation elongation factor eEF1A2. Thus, metarrestin represents a potential therapeutic approach for the treatment of metastatic cancer. Conclusion: PNC and nucleoli may play roles in metastatic cancer development.

doi: http://dx.doi.org/10.7124/bc.0009CA

\section{The systematic study on the epigenomics of mei- Cohesins in the norm and as Cancer-Testis proteins}

Abdelhalim Boukaba ${ }^{1}$, Qionfang $\mathrm{Wu}^{1}$, Jierong Liang ${ }^{1}$, Jian Liu ${ }^{1}$, Elena M. Pugache$\mathrm{va}^{3}$, Victor Lobanenkov ${ }^{3}$, and Alexander $\underline{\text { Strunnikov }}^{1,2}$

${ }^{1}$ Molecular Epigenetics Laboratory, Guangzhou Institutes of Biomedicine and Health, Guang- 